\title{
Amplifying Chiroptical Properties of Conjugated Polymer Thin-Film Using an Achiral Additive
}

\author{
Chidambar Kulkarni, ${ }^{\dagger}$ Stefan C. J. Meskers, ${ }^{\dagger}{ }^{\dagger}$ Anja R. A. Palmans, ${ }^{\dagger}$ (๑) and E. W. Meijer* ${ }^{\dagger}{ }^{\dagger}$ \\ ${ }^{\dagger}$ Laboratory of Macromolecular and Organic Chemistry and Institute for Complex Molecular Systems, Eindhoven University of \\ Technology, P.O. Box 513, Eindhoven 5600 MB, The Netherlands
}

\section{Supporting Information}

ABSTRACT: Chiral conjugated polymers bearing enantiopure side chains offer the possibility to harness the effect of chirality in organic electronic devices. However, its use is hampered by the low degree of circular polarization in absorption $\left(g_{\text {abs }}\right)$ in most of the conjugated polymer thin-films studied. Here we demonstrate a versatile method to significantly increase the $g_{\text {abs }}$ by using a few weight percentages of a commercially available achiral long-chain alcohol as an additive. This additive enhances the chiroptical properties in both absorption and emission by ca. 5-10 times in the thin-films. We envisage that the alcohol additive acts as a plasticizer which enhances the long-range chiral liquid crystalline ordering of the polymer chains, thereby amplifying the chiroptical properties in the thin-film. The application of this methodology to various conjugated polymers has been demonstrated.

\section{INTRODUCTION}

Conjugated semiconducting polymers form the active component of organic electronic devices such as solar cells, light-emitting diodes, and field-effect transistors. ${ }^{1-3}$ Most of the conjugated polymers studied bear achiral or branched, but racemic, side chains to provide better solubility in common organic solvents used for processing. ${ }^{4}$ On the other hand, conjugated polymers bearing enantiopure chiral side chains display new functionalities. ${ }^{5,6}$ Circularly polarized photo- and electroluminescent devices based on chiral conjugated oligomers and polymers have been studied for application in advanced display technology. ${ }^{7-10}$ Furthermore, chirality of the molecular semiconductor has been utilized to construct chiral sensors $^{11}$ and to detect circularly polarized light. ${ }^{12}$ The enantiopure molecular materials can show chiral-induced spin-selectivity, ${ }^{13}$ and thus chiral molecular semiconductors can be applied as spin-filters. ${ }^{14}$ The impact of the chirality of a semiconductor was further demonstrated by Fuchter and coworkers, where they observed over 80 -fold difference in the hole mobility of 1-aza[6]-helicene in the thin-film state between enantiopure and racemic mixture. ${ }^{15}$ Thus, it is clear that chirality of a system or chiral supramolecular organization attained through enantiomerically pure side chains can directly impact the desired functionality. ${ }^{5,6}$ In order to exploit the manifestation of chirality in semiconductor devices, it is paramount to achieve and control high degree of circular polarization (defined as $g=2 \times\left[\left(I_{\mathrm{L}}-I_{\mathrm{R}}\right) /\left(I_{\mathrm{L}}+I_{\mathrm{R}}\right)\right]$, where $I_{\mathrm{L}}$ and $I_{\mathrm{R}}$ are the intensities of left and right circularly polarized light absorbed $\left(g_{\mathrm{abs}}\right)$ or emitted $\left(g_{\mathrm{PL}}\right)$, respectively) of chiral conjugated polymer thin-films.

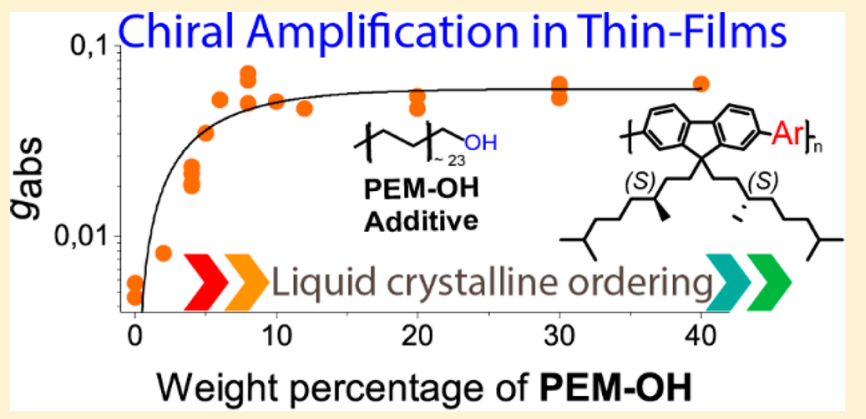

Various approaches to achieve high $g$-values $\left(g_{\mathrm{abs}}, g_{\mathrm{PL}}\right.$, and $\left.g_{\mathrm{EL}}\right)$ in molecular systems can be broadly classified into two main categories, namely, based (i) on molecular or local origin, and (ii) on long-range ordering or a nonlocal mechanism. The local origin of photo- and electroluminescence $\left(g_{\mathrm{EL}}>1\right)$ is observed for lanthanide ${ }^{16-18}$ and platinium ${ }^{19}$ complexes with chiral ligands. Since the emission originates at a molecular level, the observed $g$-values are independent of the long-range ordering in the system. Although this approach yields high $g$ values, it has a few shortcomings, namely, a low photoluminescence quantum yield, an electronically forbidden transitions in metal complexes leading to narrow emission band, and finally the structurally unique chiral ligands such as helicenes further limiting the scope of this approach.

On the other hand, Chen and co-workers have shown that chiral oligo(fluorenes) ${ }^{8,20}$ exhibit highly ordered chiral liquid crystalline phases such as cholesterics in annealed films with $g$ values $>0.1$. The cholesteric ordering has been mainly observed for poly(fluorene) homopolymers, ${ }^{21,22}$ copolymer, $^{23-26}$ and poly ( $p$-phenyleneethynylene) $\mathrm{s}^{27}$ which exhibited $g$-values ranging from 0.1 to 1 in annealed thin-films. Unlike the metal complexes discussed previously, the chiral poly(fluorene) $\mathrm{s}^{28,23}$ and poly(thiophene) $\mathrm{s}^{29}$ show a strong filmthickness dependence of $g_{a b s}$, indicating that the long-range cholesteric ordering dictates the $g$-value in such systems. Chiral poly(fluorene-alt-benzothiadiazole) (c-PFBT) has been shown

Received: May 23, 2018

Revised: July 16, 2018

Published: July 26, 2018 
a

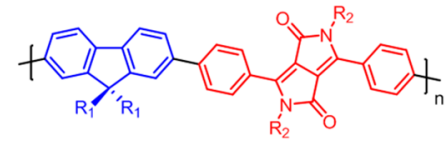

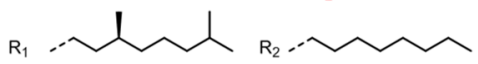

$R_{1}$ b

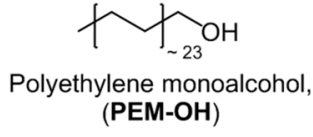

$M_{\mathrm{n}}=700 \mathrm{~g} \mathrm{~mol}^{-1}$

PDI $\left(M_{\mathrm{w}} / M_{\mathrm{n}}\right)=1.1$

$T_{\mathrm{m}}=108-110^{\circ} \mathrm{C}$

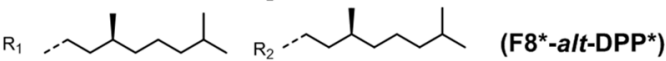
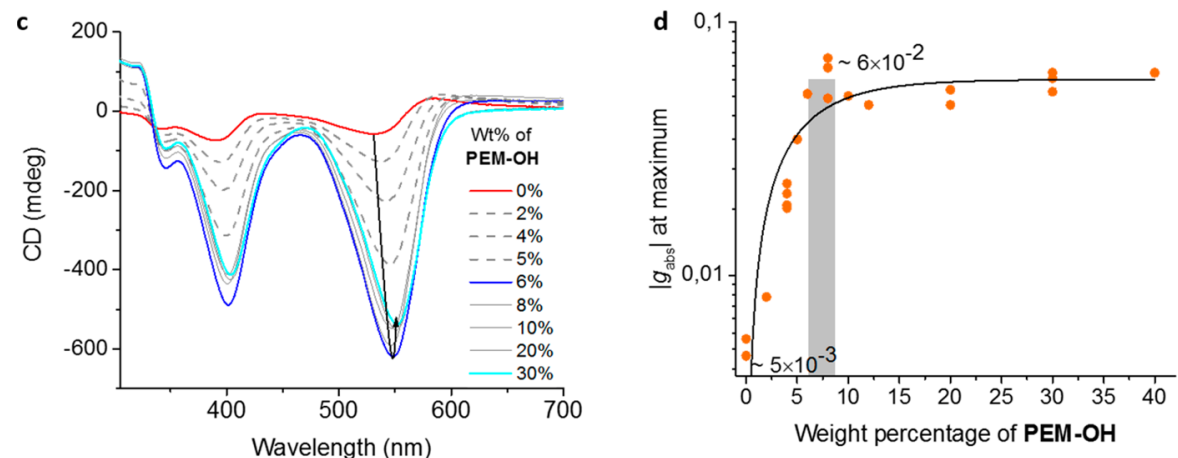

Figure 1. Enhancing chiroptical properties with an additive. (a) Structure of the fluorene copolymers investigated in this study. Asterisk sign indicates the position of the chiral side chains. (b) Structure and important physical properties of the achiral polyethylene monoalcohol (PEM$\mathbf{O H}$ ) used as a processing additive. (c) CD spectra of F8*-alt-DPP films embedded with different weight percentage of PEM-OH at $20{ }^{\circ} \mathrm{C}$. The films were annealed at $280^{\circ} \mathrm{C}$ for $15 \mathrm{~min}$ and cooled down to room temperature before measuring $\mathrm{CD}$ spectra. Arrow indicates spectral changes with increasing weight percentage of PEM-OH. (d) $\left|g_{a b s}\right|$ at CD maximum as a function of weight percentage of PEM-OH based on CD spectra shown in (c). The solid black line is a guide to the eye to indicate the trend in $\left|g_{\text {abs }}\right|$. The gray bar indicates the percentage around which maximum enhancement is observed. Typical film thickness $=120 \pm 20 \mathrm{~nm}$.

to form a multidomain cholesteric organization with high $g$ values, ${ }^{23,30}$ and this has been utilized to achieve highly circularly polarized OLEDs. ${ }^{9}$ To enhance the chiral organization of c-PFBT through cholesteric ordering and plasmonic effect, Prasad and co-workers have used gold nanoparticles (diameter 4-10 nm) as additive to c-PFBT polymer, and annealing the nanocomposite films resulted in enhanced circular dichroism effect. ${ }^{31}$ Further, an alternative route to induce cholesteric organization and high $g$-values to conjugated polymers was successfully demonstrated by the addition of a chiral dopant with high helical twisting power to an achiral conjugated polymer such as poly $\left(9,9^{\prime}\right.$-dioctylfluorene-altbenzothiadiazole) (F8BT). ${ }^{32,33}$ In spite of the abovementioned efforts, a vast number of enantiopure chiral conjugated polymers exhibit no apparent cholesteric organization, thus impeding their applications.

Here we have developed a generally applicable and facile method to significantly enhance the chiroptical properties of conjugated polymer thin-films. Our approach uses a cheap, commercially available, and achiral additive to improve the chiral liquid crystalline ordering of enantiopure conjugated polymer thin-films, thus leading to significantly enhanced chiroptical properties. To study the effect of the additive, we have chosen the chiral analogues of a known archetypical redemitting diketopyrrolopyrrole (DPP) alternating fluorene copolymer. ${ }^{34}$ First, we show the effect of the additive on the chiroptical and photophysical properties of the polymer. Then, we shed light on the influence of the additive on the mesoscopic organization of the polymer, and finally we conclude by showing the general applicability of the approach to other conjugated polymers.

\section{RESULTS AND DISCUSSION}

All the three fluorene-alt-DPP polymers (Figure 1a) were synthesized by Suzuki-polycondensation and characterized using ${ }^{1} \mathrm{H}-,{ }^{13} \mathrm{C}$ NMR, and GPC (See Supporting Information Figures S1-S18). All polymers showed good thermal stability without any degradation until $300{ }^{\circ} \mathrm{C}$ (Figure S19). Further thermal analysis by differential scanning calorimetry (DSC) showed that all polymers exhibit only a weak endothermic transition at high temperatures, without any discernible glasstransition temperature (Figure S20). Polarized optical microscopy (POM) images of F8*-alt-DPP polymer recorded under cross-polarizer showed that around the weak endothermic transition observed in DSC, the polymer becomes more fluidic, and the birefringence increases and moreover the birefringence is retained on further cooling the sample to room temperature (Figure S21). Thus, based on DSC and POM results, we designate the temperature at which polymer transitions from a less ordered amorphous to ordered, birefringent phase as $T_{\text {trans. }}$. Due to the rather weak and broad $T_{\text {trans }}$ transition, all the polymers thin-films were annealed at higher temperature $\left(280{ }^{\circ} \mathrm{C}\right)$ to ensure the complete transition. The thermal and molecular weight distribution of the polymers are presented in Table S1. Most of the studies here will be focused on F8*-alt-DPP polymer, where the asterisk sign indicates the position of the chiral unit.

Enhancing Chiroptical Properties of F8*-alt-DPP Thin-Films with an Additive. The pristine films $(\sim 120 \pm$ $20 \mathrm{~nm}$ ) of F8*-alt-DPP spin-coated from a chloroform solution on glass substrate did not exhibit any Cotton effect (Figure S22a). Upon annealing, a clear Cotton effect was observed, and the maximum effect was seen when annealed at $280{ }^{\circ} \mathrm{C}$ (Figure S22a), beyond the $T_{\text {trans }}$ of the polymer $(\sim 240$ $\left.{ }^{\circ} \mathrm{C}\right)$. The $g_{\text {abs }}$ was found to be $-5 \times 10^{-3}$ (at $540 \mathrm{~nm}$ ), which is 

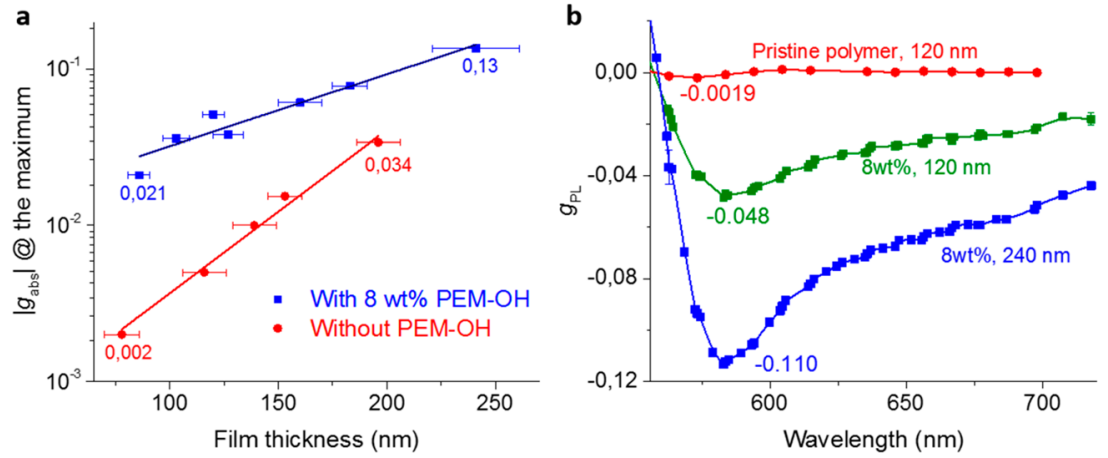

Figure 2. (a) Thickness dependence of $\left|g_{\text {abs }}\right|$ for F8*-alt-DPP annealed thin-films without and with (8 wt \%) PEM-OH. The solid lines is a guide to the eye to indicate the trend. Error bars in the film thickness indicate the thickness distribution on different parts of the film. (b) Degree of circular polarization in photoluminescence $\left(g_{\mathrm{PL}}\right)$ without and with $(8 \mathrm{wt} \%)$ of PEM-OH when excited at $356 \mathrm{~nm}$. The weight percentage of the PEM-OH and film thickness are mentioned in (b). All the films were annealed at $280{ }^{\circ} \mathrm{C}$ for $15 \mathrm{~min}$, and the spectra are recorded at $20{ }^{\circ} \mathrm{C}$.

in similar range as observed for most of the conjugated polymers in thin-films. The low magnitude of the $g_{\text {abs }}$ can be explained by the local chiral ordering of the polymer chains.

In order to enhance the chiroptical properties of $\mathbf{F} 8 *$-altDPP in the thin-film, here we have chosen the commercially available alcohol, namely, polyethylene monoalcohol (PEM$\mathrm{OH})$ with an average 48 methylene units and a $T_{\mathrm{m}}$ of $108-110$ ${ }^{\circ} \mathrm{C}$ as an additive (Figure $1 \mathrm{~b}$ ). First we look at the effect of the additive on the Cotton effect at a constant film thickness $(\sim 120 \pm 20 \mathrm{~nm})$. The PEM-OH and F8*-alt-DPP were mixed in a good solvent and spin-coated on glass substrates and annealed beyond the $T_{\text {trans }}$ and $T_{\mathrm{m}}$ of both F8*-alt-DPP and PEM-OH, respectively, and finally the CD spectra of thinfilms were recorded at $20{ }^{\circ} \mathrm{C}$ (see the Methods section for detailed procedure). The $\mathrm{CD}$ spectra show a pronounced enhancement in Cotton effect with addition of small amount (2-6 wt \%) of PEM-OH. The sign of the Cotton effect is unaffected, and only the magnitude increases with addition of PEM-OH. The enhancement in magnitude of the Cotton effect saturates around 6-8 wt \%, and with further increase in the amount of PEM-OH, a small decrease in the magnitude of the Cotton effect was observed (Figure 1c). We noticed a 15$20 \mathrm{~nm}$ red-shift of the CD maximum going from 0 to $40 \mathrm{wt} \%$ of PEM-OH. To quantify the enhancement in chiroptical properties, we study the evolution of $g_{\text {abs }}$ with addition of PEM-OH. The $g_{\text {abs }}$ changes from $-5 \times 10^{-3}$ for pristine polymer film to $-6 \times 10^{-2}$ for 8 wt $\%$ of PEM-OH blended films (Figure 1d). The $g_{\text {abs }}$ remained $-5 \pm 1 \times 10^{-2}$ with addition of higher weight percentage of PEM-OH. Linear dichroism spectra on films with different weight percentage of PEM-OH showed negligible anisotropy in the films (Figure S23), indicating that such artifacts do not contribute significantly to the observed CD signals. For all the different weight percentages of $\mathbf{P E M - O H}$, the UV-vis absorption spectra of the polymer remain unaltered in the thin-film (Figure S24). Similarly, both the steady-state and timeresolved photoluminescence spectra of F8*-alt-DPP are unaltered by the addition of PEM-OH (Figure S25), suggesting no electronic perturbation of the F8*-alt-DPP on addition of PEM-OH.

As it has been previously shown that $g_{\text {abs }}$ strongly depends on the film thickness for fluorene-based polymers, ${ }^{28}$ we have looked into the film-thickness dependence of $g_{\text {abs }}$ for F ${ }^{*}$-altDPP both without and with PEM-OH. Annealed thin-films of F8*-alt-DPP (without PEM-OH) show an increase in $g_{\text {abs }}$ from $-0.002(80 \mathrm{~nm})$ to $-0.034(200 \pm 10 \mathrm{~nm})$ with an increase in film thickness (Figure 2a). Further, by combining the effect of PEM-OH (8 wt \%) with the film-thickness dependence we could observe significant enhancement in $g_{\text {abs }}$, and a maximum $g_{\text {abs }}$ of -0.13 could be achieved for a film thickness of $240 \pm 20 \mathrm{~nm}$ (Figure 2a). This indicates that both the effect of PEM-OH and film-thickness dependence operate synergistically to achieve high $\left|g_{\text {abs }}\right|(>0.1)$ in annealed films.

We have further investigated the influence of the PEM-OH additive and film thickness on the degree of circular polarization in photoluminescence $\left(g_{\mathrm{PL}}\right)$ for $\mathbf{F} 8 *$-alt-DPP. Pristine annealed F8*-alt-DPP film (thickness of $120 \mathrm{~nm}$ ) shows a $g_{\mathrm{PL}}$ of -0.0019 (at $573 \mathrm{~nm}$ ). For a film of similar thickness with 8 wt \% of PEM-OH, a $g_{\mathrm{PL}}$ of -0.048 (at 583 $\mathrm{nm}$ ) was observed, showing $>25$ times increase in $g_{\mathrm{PL}}$ on addition of PEM-OH. Combining both the effects of adding PEM-OH (8 wt \%) and film thickness $(240 \pm 20 \mathrm{~nm}), g_{\mathrm{PL}}$ of -0.11 (at $583 \mathrm{~nm}$ ) was observed with an enhancement in $g_{\mathrm{PL}}$ of $>50$ times compared to the pristine annealed films of F8*alt-DPP. Thus, the PEM-OH additive can lead to significant enhancement in chiroptical properties ( $g_{\text {abs }}$ and $\left.g_{\mathrm{PL}}\right)$.

To understand the origin of the enhancement on addition of PEM-OH, we first studied if the alcohol group of PEM-OH is really necessary for amplification of chiroptical properties in the thin-film. In place of PEM-OH, a linear chain alkane with 42 methylene units $\left(\mathrm{C}_{44} \mathrm{H}_{90}\right.$, tetratetracontane $)$, similar to the number of methylene units in PEM-OH, was used as an additive. The CD spectra of the pristine annealed film and with 7 wt \% of tetratetracontane were identical (Figure S26a), indicating no enhancement in $g_{\text {abs }}$ on addition of tetratetracontane. It is to be noted that with PEM-OH at similar weight percentage $(6-8 \mathrm{wt} \%)$, the maximum enhancement was already observed. However, with 20 wt \% of tetratetracontane the $g_{\text {abs }}$ increased to -0.02 , an enhancement of $<2$ times compared to the pristine films (Figure S26c). This suggests that alcohol group on the long-chain alkane is important in achieving high enhancement factor. Further, we have also looked into the role of polymer self-assembly in alcohol as solvent and its relation to the enhancement in chiroptical properties in thin-film. First we studied F8*-altDPP, which showed significantly high $g_{\text {abs }}(\sim-0.02)$ when self-assembled in dilute $n$-butanol as solvent (Figure S27), and it was also observed that addition of PEM-OH resulted in improved $g_{\text {abs }}$ in annealed thin-film (Figure 1c). However, F8alt-DPP*, a structural isomer of F8*-alt-DPP with chiral side 

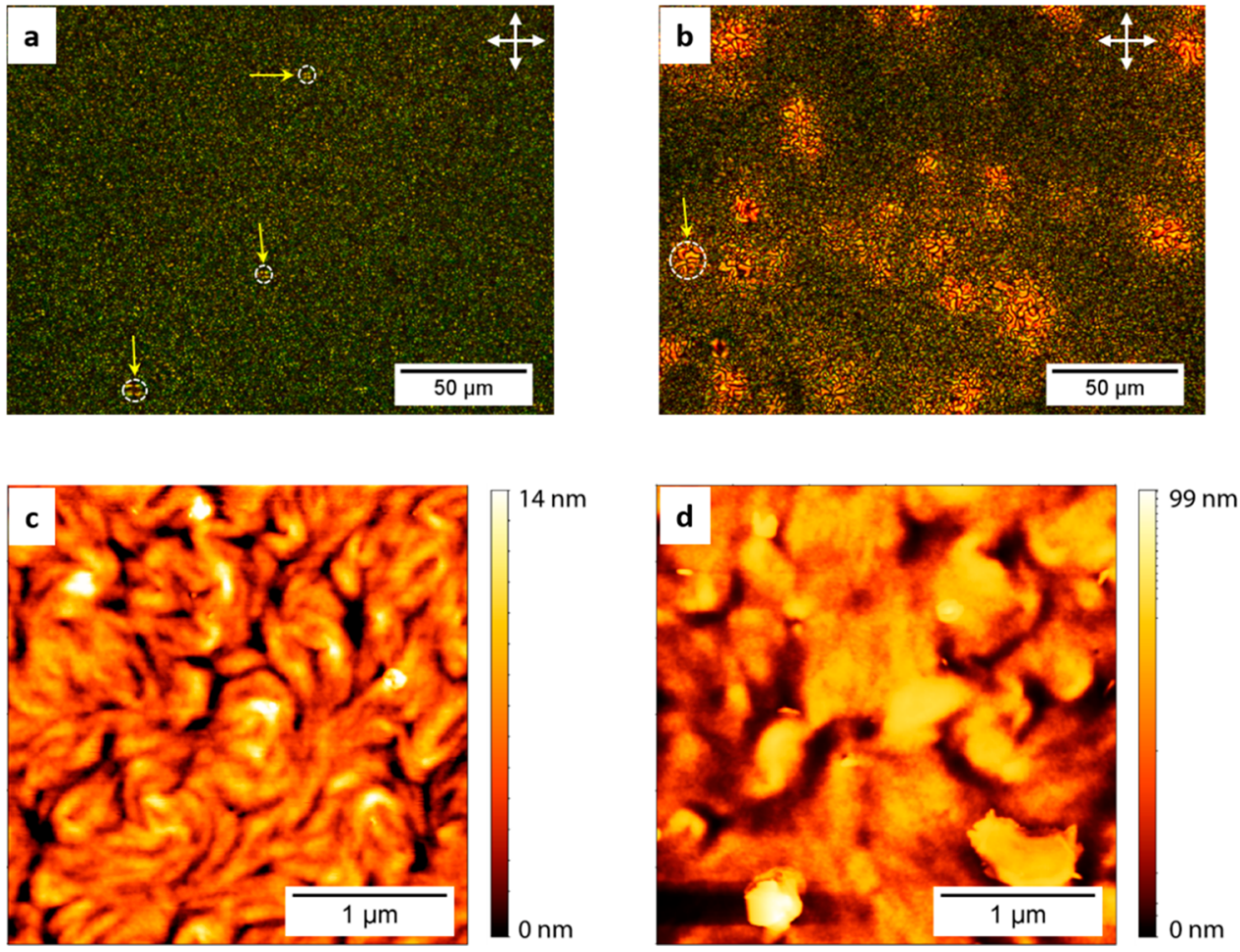

Figure 3. Morphological characterization of the effect of PEM-OH on F8*-alt-DPP annealed thin-films. (a) and (b) Polarized optical microscopy image of F8*-alt-DPP annealed films without and with $10 \mathrm{wt} \%$ of PEM-OH, respectively, under crossed polarizers. The white circles indicate regions in which a nematic like texture is observed. Note that the size of regions is much larger in (b) compared to (a). The thickness of film for POM images was $165 \pm 10 \mathrm{~nm}$. (c) and (d) Tapping mode atomic force microscopy height images of F8*-alt-DPP annealed films without and with 10 wt \% PEM-OH, respectively. Note that the scale bar for both $(\mathrm{c})$ and $(\mathrm{d})$ is the same $(1 \mu \mathrm{m})$. The film thickness for AFM studies was $\sim 120 \mathrm{~nm}$.

chains on the DPP unit, did not show any Cotton effect (helical organization) in $n$-decanol (Figure S28a), and also the annealed thin-films of F8-alt-DPP* with PEM-OH additive showed low $g_{\text {abs }}$ of $10^{-3}$ (Figure S28b, c). Where the solution experiments give evidence for the presence of a chiral packing in nanosized aggregates, the thin-film experiments show the presence of macroscopic chiral liquid crystalline ordering. Apparently, the need for a nanoscopic local arrangement is amplified macroscopically.

Morphological Characterization of Pristine and PEM$\mathrm{OH}$ Blended Films. Having established the importance of polymer self-assembly in alcohols as solvent and the role of alcohol group of PEM-OH in enhancement of chiroptical properties, we further investigated the interaction of PEM-OH with F8*-alt-DPP polymer chains. The FT-IR spectra of F8*alt-DPP thin-films $(\sim 120 \mathrm{~nm})$ without and with $5-20 \mathrm{wt} \%$ of PEM-OH showed no clear shifts in both the $\mathrm{C}=\mathrm{O}$ stretching of the DPP units and the $\mathrm{C}-\mathrm{H}$ stretching of the alkyl chains (Figure S29), suggesting no chemical interactions between F8*-alt-DPP and PEM-OH. The effect of the PEM-OH on mesoscopic organization of F8*-alt-DPP was further investigated by polarized optical microscopy (POM). POM images of pristine annealed films $(\sim 165 \pm 10 \mathrm{~nm})$ show birefringence without a clear texture. However, on closer examination we see small features $(3-6 \mu \mathrm{m})$ reminiscent of nematic ordering (Figure 3a). On the other hand, the 10 wt \% PEM-OH blended films shows clear birefringence with large domains $(10-20 \mu \mathrm{m})$ present through most part of the film (Figure $3 \mathrm{~b})$. This suggests that, on addition of PEM-OH, the chiral liquid crystalline ordering in the sample gets enhanced. The effect of PEM-OH on surface topography was studied through tapping-mode atomic force microscopy (AFM). The AFM height images of pristine annealed F8*-alt-DPP films shows a network of fibers with a very smooth surface $( \pm 14 \mathrm{~nm}$, Figure $3 \mathrm{c})$. However, the $10 \mathrm{wt} \%$ blended films show platelet like topography with an increased surface roughness of $80-100 \mathrm{~nm}$ (Figure 3d). The lack of fibrillary morphology indicates marked difference between the pristine and PEM-OH blended films. Further differential scanning calorimetric (DSC) studies were carried out to study the bulk phase behavior of polymer on addition of PEM-OH. Pristine polymer shows weak transition $\left(T_{\text {trans }}\right)$ around $230-240{ }^{\circ} \mathrm{C}$ (Figure S30). With 12.5 wt \% of PEM-OH embedded into the polymers, first the melting of PEM-OH was observed at $108-112{ }^{\circ} \mathrm{C}$, and with further heating near the $T_{\text {trans }}$ of $\mathbf{F} 8 \boldsymbol{*}^{*}$-alt-DPP a clear exothermic transition was observed (Figure S30). This exothermic peak (cold crystallization) on the heating run indicates ordering of polymer and PEM-OH mixture near the $T_{\text {trans }}$ of F8*-alt-DPP.

In order to study this ordering, bulk wide-angle X-ray scattering (WAXS) experiments were performed. The WAXS profile of annealed F8*-alt-DPP shows a principal peak at 0.35 $\AA^{-1}$ corresponding to a distance of $17.5 \AA$ which matches well with the distance between the fully elongated chiral chains on the fluorene (Figure S31a, b). In addition to the principal peak, other weak peaks at 0.68 and $1.21 \AA^{-1}$ and a broad halo centered at $1.38 \AA^{-1}$ were observed. These features suggests that F8*-alt-DPP exhibits a semicrystalline organization, and this is also consistent with the disordered fibrillary morphology observed in AFM (Figure 3c). On addition of PEM-OH, only 


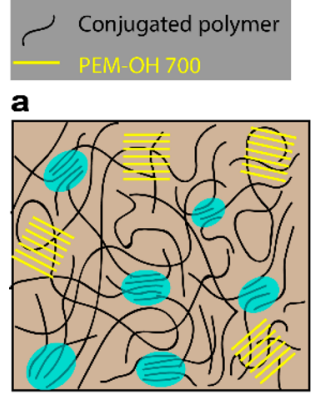

At room temperature

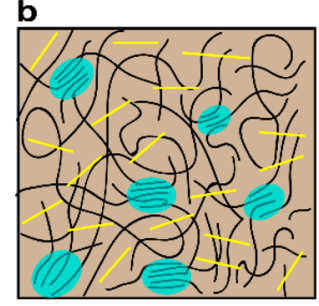

At $T_{\mathrm{m}}\left(108-110^{\circ} \mathrm{C}\right)$ of PEM-OH

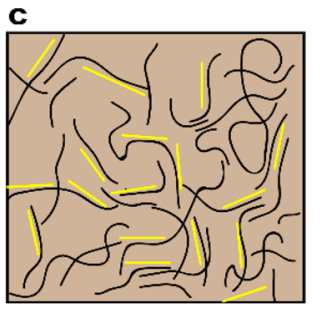

At $T_{\text {trans }} / T_{\mathrm{m}}$ of polymer

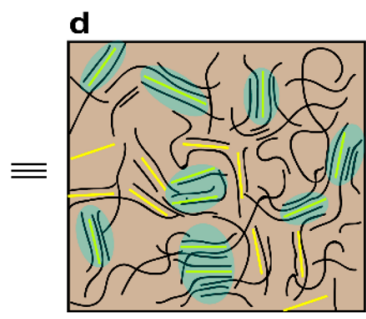

Cold crystallization

Temperature

Figure 4. Schematic illustration of the influence of PEM-OH on the morphology of the conjugated polymer. The glass substrate is depicted in gray, the black lines represent polymer chains, the yellow lines indicate $\mathbf{P E M}-\mathbf{O H}$, and the cyan regions illustrate the semiordered regions in the sample. (a) Shows the physical mixture of crystalline PEM-OH and semicrystalline polymer at room temperature. (b) Around the melting point of PEM$\mathbf{O H}$, the crystallites of PEM-OH break down, and the polymer does not undergo any changes. On furthering heating until $T_{\text {trans }}$ or $T_{\mathrm{m}}$ of polymer, both polymer and PEM-OH mix (c) and instantaneously undergo cold-crystallization (d) leading to ordered regions containing PEM-OH (the cyan regions in (d)).

minor changes were observed in the WAXS profile, suggesting that the semicrystalline nature of $\mathbf{F}^{*} *$-alt-DPP is maintained (Figure S31c). It is worth noting that the liquid crystalline ordering observed in POM on addition of PEM-OH is at a much larger length scale (few micrometers) compared to local ordering (nanometer scale) probed using WAXS measurements.

Based on DSC, POM, and AFM studies, the structural evolution of the F8*-alt-DPP on addition of PEM-OH can be visualized as shown in Figure 4. At room temperature (either in thin-film or bulk), both PEM-OH and the semicrystalline F8*-alt-DPP exist as physical mixture (Figure 4a). On heating beyond the melting point of PEM-OH, it melts and blends into the matrix of F8*-alt-DPP (Figure 4b). On further heating near the $T_{\text {trans }}$ of the polymer, the polymer softens or in other words the semicrystalline regions in the polymer melt (Figure 4c), and it now interacts with the molten PEM-OH leading to cold crystallization or ordering into a nematic-like phase (Figure 4d). Thus, the PEM-OH enhances the chiroptical properties in the thin-film by improving the chiral liquid crystalline ordering of the polymer chains without a direct chemical interaction with the polymer chains.

Generality of the PEM-OH Methodology. Finally, we have looked into the general applicability of the PEM-OH methodology to enhance chiroptical properties in other $\pi$ conjugated polymers. The polymers we have chosen are the fully chiral F8*-alt-DPP*, c-PFBT, and chiral poly(thiophene) (PBMBT) (Figure 1a and 5a). F8*-alt-DPP* and c-PFBT polymers self-assemble in $n$-decanol with $g_{\text {abs }}$ of 0.008 and -0.07 , respectively (Figure S32). Chiral poly(thiophene) (PBMBT) has also been observed to show significantly high $g_{\text {abs }}(\sim 0.08)$ in $n$-decanol as solvent at low concentration. ${ }^{35,36}$ Further, the PEM-OH embedded films indeed show an enhancement of 10 and 5 times for F8*-alt-DPP* and cPFBT, respectively (see Figure S33, Figure S34, Figure 5b, and Table 1). Annealed PBMBT films blended with different weight percentage of PEM-OH also showed improved chiroptical properties (Figure S35, Figure 5b), but the maximum $g_{\text {abs }}$ was observed around $30 \mathrm{wt} \%$ of the additive (Table 1).
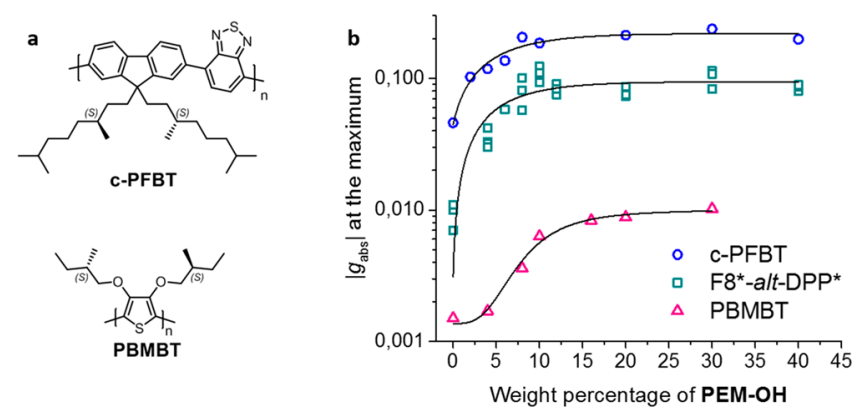

Figure 5. Application of PEM-OH addition strategy to other $\pi$ conjugated polymers. (a) Chemical structure of c-PFBT and PBMBT. (b) Evolution of $\left|g_{a b s}\right|$ at the maximum of CD effect in annealed thin-films as a function of different weight percentage of PEM-OH. The black solid is a guide to the eye. c-PFBT, PBMBT, and F $^{*}$-alt-DPP* films were annealed at 150,170 , and $280{ }^{\circ} \mathrm{C}$, respectively, for $15 \mathrm{~min}$ and cooled down. Thicknesses of c-PFBT, PBMBT, and F8*alt-DPP* films were $90 \pm 10,80 \pm 20$, and $120 \pm$ $10 \mathrm{~nm}$, respectively. All the CD spectra were recorded at $20{ }^{\circ} \mathrm{C}$.

Further the PBMBT films annealed at $120^{\circ} \mathrm{C}$ (below the $T_{\mathrm{m}}$ $\sim 160{ }^{\circ} \mathrm{C}$ of polymer) did not show any enhancement in Cotton effect (Figure S36). However, when the films were annealed at $170^{\circ} \mathrm{C}$, that is, beyond the $T_{\mathrm{m}}$ of both the PEMOH $\left(108-110{ }^{\circ} \mathrm{C}\right)$ and PBMBT $\left(\sim 160{ }^{\circ} \mathrm{C}\right)$, the $g_{\text {abs }}$ was significantly enhanced (Figure $5 \mathrm{~b}$ and Figure S35). This indicates that annealing beyond the $T_{\mathrm{m}}$ of both PEM-OH and the polymer is necessary for them to undergo intermixing and leading to more ordered organization. DSC thermogram of PBMBT with 4 wt \% of PEM-OH showed improved crystallization compared to the pristine polymer (Figure S37). Similarly, F8*-alt-DPP* also showed a strong cold crystallization with $27 \mathrm{wt} \%$ of PEM-OH at $\sim 230{ }^{\circ} \mathrm{C}\left(T_{\text {trans }}\right.$ of the polymer, Figure S38). These observations are similar to that seen for F8*-alt-DPP, indicating that a similar mechanism of enhancement is operative for these polymers as well.

Furthermore, the PEM-OH blended films of c-PFBT show $g_{\mathrm{PL}}$ reaching -0.2 to -0.3 at higher weight percentage of PEMOH (Figure S39). It is noteworthy that c-PFBT was previously shown to exhibit high $g_{\text {abs }}(\sim 0.15)$ at an optimum of molecular weight. ${ }^{23}$ However, in the present study we have utilized the as 
Table 1. Comparison of Chiroptical Properties of Different Polymers with and without PEM-OH

\begin{tabular}{|c|c|c|c|c|c|}
\hline polymer & $\begin{array}{l}\mathrm{g}_{\mathrm{abs}} \text { for pristine } \\
\text { films }{ }^{a}\left(\mathrm{~g}_{\mathrm{abs}, \text { prist }}\right)\end{array}$ & $\begin{array}{l}\max . g_{\text {abs }} \text { for PEM-OH blended films } s^{a} \\
\left(g_{\text {abs, }} \text { PEM-OH }\right)(\text { wt \% of PEM-OH })^{b}\end{array}$ & $\begin{array}{l}\text { enhancement factor } \\
\left(g_{\text {abs,PEM-OH }} / g_{\text {abs,prist }}\right)\end{array}$ & $\begin{array}{l}g_{\mathrm{PL}} \text { for } \\
\text { pristine films }\end{array}$ & $\begin{array}{l}\text { max. } g_{\text {PL }} \text { for PEM-OH } \\
\text { blended films }{ }^{a}\end{array}$ \\
\hline F8*-alt-DPP & $-5 \times 10^{-3}$ & $-6 \times 10^{-2}(8$ wt $\%)$ & 12 & $-9 \times 10^{-3}$ & $5 \times 10^{-2}$ \\
\hline F8*-alt-DPP* & $-8 \times 10^{-2}$ & $-1 \times 10^{-1}(10$ wt $\%)$ & 12.5 & N.D. & N.D. \\
\hline c-PFBT & $-4 \times 10^{-2}$ & $-2 \times 10^{-1}(10$ wt $\%)$ & 5 & $-3 \times 10^{-2}$ & $2.4 \times 10^{-1}$ \\
\hline PBMBT & $-1 \times 10^{-3}$ & $-9 \times 10^{-2}(30 \mathrm{wt} \%)$ & $6-8$ & N.D. & N.D. \\
\hline
\end{tabular}

${ }^{a}$ Annealed films. ${ }^{b}$ The values in the parentheses indicate the percentage were maximum $g_{\text {abs }}$ was observed, N.D.: not determined.

synthesized polydisperse sample without fractionation and could still achieve higher $g_{\text {abs }}(>0.2)$ than those observed for the optimum molecular weight at a given film thickness $(\sim 80$ $\mathrm{nm})$. Moreover, Fuchter and co-workers ${ }^{33}$ used $53 \%$ of 1 aza[6] helicene to achieve a $g_{\text {abs }}$ of 0.2 for achiral F8BT, whereas we could reach similar $g_{\text {abs }}$ values at only $8-10 \mathrm{wt} \%$ of PEM-OH for c-PFBT. It was also observed that at low loading of PEM-OH $(<10 \mathrm{wt} \%)$ the films were macroscopically uniform without affecting any of the photophysical properties of the pristine polymers.

\section{OUTLOOK AND CONCLUSION}

Here we have developed a method to improve the chiroptical properties in the thin-films of enantiopure conjugated polymers using a commercially available achiral long-chain alcohol (PEM-OH) as an additive. The enhancement in chiroptical properties is ca. 5-10 times in the annealed thinfilms and is applicable to a broad range of enantiopure conjugated polymers. Based on microscopic studies, we attribute the enhancement in chiroptical properties to improved chiral liquid crystalline ordering of polymer chains on addition of PEM-OH. We envisage that the PEM-OH acts as a supramolecular plasticizer which aids in providing increased mobility to the polymer chains beyond $T_{\text {trans }}$ or $T_{\mathrm{m}}$, thereby improving their chiral liquid crystalline organization. PEM-OH can be viewed as a processing agent to enhance chiroptical properties, analogues to the alkane di(thiol/iodide) which are widely used additives to enhance the efficiency of organic solar cells. ${ }^{37,38}$

We have identified the following criteria to be crucial for the PEM-OH methodology to work: (i) the chiral self-assembly of the enantiopure conjugated polymers in alcohols as solvent is a prerequisite to using the PEM-OH method in the thin-film, and (ii) in principle this methodology could work with other long-chain alcohols with appropriate thermal behavior $\left(T_{\mathrm{m} \text {, alcohol }}<T_{\text {trans, polymer }} / T_{\mathrm{m} \text {, polymer }}\right.$ and that the alcohol additive remains in the molten state at the $T_{\text {trans }}$ or $T_{\mathrm{m}}$ of the polymer). Since PEM-OH is a disperse polymer, it would be interesting to investigate the effect of the dispersity of the long-chain alcohol additive on the chiroptical enhancement. We anticipate that this new class of UV-vis-NIR transparent long-chain alcohol additives which does not affect the photophysical properties of the conjugated polymers will pave the way for harnessing the chirality of enantiopure conjugated polymers for various applications.

\section{METHODS}

Sample Preparation for PEM-OH Blended Films. A stock solution of PEM-OH at a concentration of $1 \mathrm{mg} / \mathrm{mL}$ was prepared by dissolving the required amount of freshly powdered PEM-OH (as obtained from Sigma-Aldrich) in chloroform as the solvent in a vial with PTFE seal cap (Note 1). The sealed vial containing PEM-OH and chloroform was heated at $70{ }^{\circ} \mathrm{C}$ (oil bath temp) for $2 \mathrm{~h}$ to ensure complete dissolution of PEM-OH in chloroform. The vial was further sonicated for $5 \mathrm{~min}$ and heated with a heat gun. Separate vials (1.5 $\mathrm{mL}$ ) containing $\sim 1.5-1.7 \mathrm{mg}$ of the polymer were charged with different amounts of the semihot PEM-OH stock solution (Note 2). The chloroform from each of the sample vials was evaporated by gently blowing $\mathrm{N}_{2}$ gas and finally heating with heat gun to ensure complete removal of the solvent. To each of the sample vials, fresh chloroform was added such that the concentration of the sample with respect to the polymer was $15 \mathrm{mg} / \mathrm{mL}$ (Note 3). These vials were further heated on an oil bath at $70{ }^{\circ} \mathrm{C}$ for $2 \mathrm{~h}$ to ensure the homogeneous mixing of the polymer and PEM-OH. The samples once cooled down to room temperature were spin-coated (2000 rpm, $60 \mathrm{~s}$, maximum acceleration) on clean glass slides. The slides were further annealed beyond the $T_{\text {trans }}$ or $T_{\mathrm{m}}$ of the polymers in a glovebox for $15 \mathrm{~min}$. All the spectra are recorded at room temperature $\left(20^{\circ} \mathrm{C}\right)$.

Note 1: It is important to freshly powder the commercially available PEM-OH pallets instead of using powdered and stored PEM-OH. The extent of chirality enhancement was found to be low with stored powder. Also it was observed that powdered and stored PEM-OH was difficult to dissolve in chloroform using the abovedescribed procedure. We think it is mostly due to the hygroscopic nature of the PEM-OH, which might result in powdered sample adsorbing water and making it difficult to dissolve in chloroform and consequently affecting the enhancement factor.

Note 2: By semihot we mean at a temperature of around 30-40 ${ }^{\circ} \mathrm{C}$. By allowing the PEM-OH stock solution to cool down to room temperature, we saw a white precipitate. Thus, PEM-OH may not be completely soluble in chloroform at room temperature.

Note 3: The concentration was with respect to the amount of polymer taken and not with respect to the total amount of the material (polymer + PEM-OH). This is because, by considering the concentration with respect to the total material (polymer + PEM$\mathbf{O H}$ ), the magnitude of Cotton effect decreases at higher weight percentage of PEM-OH due to lowering of polymer concentration.

\section{ASSOCIATED CONTENT}

\section{Supporting Information}

The Supporting Information is available free of charge on the ACS Publications website at DOI: 10.1021/acs.macromol.8b01077.

Synthesis and detailed characterization of the polymers; additional CD, UV-vis, and PL spectra of the polymers; DSC thermograms, POM images, $\mathrm{X}$-ray scattering data, comparison of $\mathrm{CD}$ and ellipsometry data to calculate $g_{\text {abs }}$ (PDF)

\section{AUTHOR INFORMATION}

\section{Corresponding Author}

*E-mail: e.w.meijer@tue.nl.

ORCID $\odot$

Chidambar Kulkarni: 0000-0001-8342-9256

Stefan C. J. Meskers: 0000-0001-9236-591X

Anja R. A. Palmans: 0000-0002-7201-1548

E. W. Meijer: 0000-0003-4126-7492

Notes

The authors declare no competing financial interest. 


\section{ACKNOWLEDGMENTS}

We acknowledge financial support from NWO (TOP-PUNT Grant 10018944) and the Dutch Ministry of Education, Culture, and Science (Gravitation program 024.001.035). C.K. is thankful for the Marie Skłodowska-Curie postdoctoral fellowship (704830) for financial support. We thank Dr. Bas van Genabeek for X-ray measurements and Prof. René Janssen for fruitful discussions.

\section{REFERENCES}

(1) Facchetti, A. $\pi$-Conjugated Polymers for Organic Electronics and Photovoltaic Cell Applications. Chem. Mater. 2011, 23, 733.

(2) Guo, X.; Baumgarten, M.; Müllen, K. Designing $\pi$-conjugated polymers for organic electronics. Prog. Polym. Sci. 2013, 38, 1832.

(3) Henson, Z. B.; Müllen, K.; Bazan, G. C. Design strategies for organic semiconductors beyond the molecular formula. Nat. Chem. 2012, 4, 699.

(4) Mei, J.; Bao, Z. Side Chain Engineering in Solution-Processable Conjugated Polymers. Chem. Mater. 2014, 26, 604.

(5) Brandt, J. R.; Salerno, F.; Fuchter, M. J. The added value of small-molecule chirality in technological applications. Nat. Rev. Chem. 2017, 1, 45 .

(6) Morrow, S. M.; Bissette, A. J.; Fletcher, S. P. Transmission of chirality through space and across length scales. Nat. Nanotechnol. 2017, 12, 410.

(7) Peeters, E.; Christiaans, M. P. T.; Janssen, R. A. J.; Schoo, H. F. M.; Dekkers, H. P. J. M.; Meijer, E. W. Circular Dichroism and Circular Polarization of Photoluminescence of Highly Ordered Poly $\{3,4-\operatorname{di}[(S)-2$-methylbutoxy $]$ thiophene\}. J. Am. Chem. Soc. 1997, 119, 9909.

(8) Geng, Y.; Trajkovska, A.; Culligan, S. W.; Ou, J. J.; Chen, H. M. P.; Katsis, D.; Chen, S. H. Origin of Strong Chiroptical Activities in Films of Nonafluorenes with a Varying Extent of Pendant Chirality. J. Am. Chem. Soc. 2003, 125, 14032.

(9) Di Nuzzo, D.; Kulkarni, C.; Zhao, B.; Smolinsky, E.; Tassinari, F.; Meskers, S. C. J.; Naaman, R.; Meijer, E. W.; Friend, R. H. High Circular Polarization of Electroluminescence Achieved via SelfAssembly of a Light-Emitting Chiral Conjugated Polymer into Multidomain Cholesteric Films. ACS Nano 2017, 11, 12713.

(10) Oda, M.; Nothofer, H. G.; Lieser, G.; Scherf, U.; Meskers, S. C. J.; Neher, D. Circularly Polarized Electroluminescence from LiquidCrystalline Chiral Polyfluorenes. Adv. Mater. 2000, 12, 362.

(11) Torsi, L.; Farinola, G. M.; Marinelli, F.; Tanese, M. C.; Omar, O. H.; Valli, L.; Babudri, F.; Palmisano, F.; Zambonin, P. G.; Naso, F. A sensitivity-enhanced field-effect chiral sensor. Nat. Mater. 2008, 7, 412.

(12) Yang, Y.; da Costa, R. C.; Fuchter, M. J.; Campbell, A. J. Circularly polarized light detection by a chiral organic semiconductor transistor. Nat. Photonics 2013, 7, 634.

(13) Naaman, R.; Waldeck, D. H. Chiral-Induced Spin Selectivity Effect. J. Phys. Chem. Lett. 2012, 3, 2178.

(14) Mondal, P. C.; Kantor-Uriel, N.; Mathew, S. P.; Tassinari, F.; Fontanesi, C.; Naaman, R. Chiral Conductive Polymers as Spin Filters. Adv. Mater. 2015, 27, 1924.

(15) Yang, Y.; Rice, B.; Shi, X.; Brandt, J. R.; Correa Da Costa, R.; Hedley, G. J.; Smilgies, D. M.; Frost, J. M.; Samuel, I. D. W.; OteroDe-La-Roza, A.; Johnson, E. R.; Jelfs, K. E.; Nelson, J.; Campbell, A. J.; Fuchter, M. J. Emergent Properties of an Organic Semiconductor Driven by its Molecular Chirality. ACS Nano 2017, 11, 8329.

(16) Zinna, F.; Di Bari, L. Lanthanide Circularly Polarized Luminescence: Bases and Applications. Chirality 2015, 27, 1.

(17) Zinna, F.; Giovanella, U.; Bari, L. Di. Highly Circularly Polarized Electroluminescence from a Chiral Europium Complex. Adv. Mater. 2015, 27, 1791.

(18) Zinna, F.; Pasini, M.; Galeotti, F.; Botta, C.; Di Bari, L.; Giovanella, U. Design of Lanthanide-Based OLEDs with Remarkable Circularly Polarized Electroluminescence. Adv. Funct. Mater. 2017, 27, 1603719.
(19) Brandt, J. R.; Wang, X.; Yang, Y.; Campbell, A. J.; Fuchter, M. J. Circularly Polarized Phosphorescent Electroluminescence with a High Dissymmetry Factor from PHOLEDs Based on a Platinahelicene. J. Am. Chem. Soc. 2016, 138, 9743.

(20) Geng, Y.; Trajkovska, A.; Katsis, D.; Ou, J. J.; Culligan, S. W.; Chen, S. H. Synthesis, Characterization, and Optical Properties of Monodisperse Chiral Oligofluorenes. J. Am. Chem. Soc. 2002, 124, 8337.

(21) Oda, M.; Nothofer, H. G.; Scherf, U.; Šunjić, V.; Richter, D.; Regenstein, W.; Neher, D. Chiroptical Properties of Chiral Substituted Polyfluorenes. Macromolecules 2002, 35, 6792.

(22) Watanabe, K.; Koyama, Y.; Suzuki, N.; Fujiki, M.; Nakano, T. Gigantic chiroptical enhancements in polyfluorene copolymers bearing bulky neomenthyl groups: importance of alternating sequences of chiral and achiral fluorene units. Polym. Chem. 2014, $5,712$.

(23) Abbel, R.; Schenning, A. P. H. J.; Meijer, E. W. Molecular Weight Optimum in the Mesoscopic Order of Chiral Fluorene (Co)polymer Films. Macromolecules 2008, 41, 7497.

(24) Lee, G. J.; Choi, E. H.; Ham, W. K.; Hwangbo, C. K.; Cho, M. J.; Choi, D. H. Circular dichroism, surface-enhanced Raman scattering, and spectroscopic ellipsometry studies of chiral polyfluorene-phenylene films. Opt. Mater. Express 2016, 6, 767.

(25) Nowacki, B.; Zanlorenzi, C.; Baev, A.; Prasad, P. N.; Akcelrud, L. Interplay between structure and chiral properties of polyfluorene derivatives. Polymer 2017, 132, 98.

(26) Cho, M. J.; Ahn, J.-S.; Kim, Y.-U.; Um, H. A.; Prasad, P. N.; Lee, G. J.; Choi, D. H. New fluorene-based chiral copolymers with unusually high optical activity in pristine and annealed thin films. RSC Adv. 2016, 6, 23879.

(27) Wilson, J. N.; Steffen, W.; McKenzie, T. G.; Lieser, G.; Oda, M.; Neher, D.; Bunz, U. H. F. Chiroptical Properties of Poly ( $p$ phenyleneethynylene) Copolymers in Thin Films: Large $g$-Values. $J$. Am. Chem. Soc. 2002, 124, 6830.

(28) Craig, M. R.; Jonkheijm, P.; Meskers, S. C. J.; Schenning, A. P. H. J.; Meijer, E. W. The Chiroptical Properties of a Thermally Annealed Film of Chiral Substituted Polyfluorene Depend on Film Thickness. Adv. Mater. 2003, 15, 1435.

(29) Vangheluwe, M.; Verbiest, T.; Koeckelberghs, G. Influence of the Substitution Pattern on the Chiroptical Properties of Regioregular Poly(3-alkoxythiophene)s. Macromolecules 2008, 41, 1041.

(30) Kulkarni, C.; Di Nuzzo, D.; Meijer, E. W.; Meskers, S. C. J. Pitch and Handedness of the Cholesteric Order in Films of a Chiral Alternating Fluorene Copolymer. J. Phys. Chem. B 2017, 121, 11520.

(31) Oh, H. S.; Liu, S.; Jee, H.; Baev, A.; Swihart, M. T.; Prasad, P. N. Chiral Poly(fluorene-alt-benzothiadiazole) (PFBT) and Nanocomposites with Gold Nanoparticles: Plasmonically and Structurally Enhanced Chirality. J. Am. Chem. Soc. 2010, 132, 17346.

(32) Lee, D.-M.; Song, J.-W.; Lee, Y.-J.; Yu, C.-J.; Kim, J.-H. Control of Circularly Polarized Electroluminescence in Induced Twist Structure of Conjugate Polymer. Adv. Mater. 2017, 29, 1700907.

(33) Yang, Y.; Da Costa, R. C.; Smilgies, D. M.; Campbell, A. J.; Fuchter, M. J. Induction of Circularly Polarized Electroluminescence from an Achiral Light-Emitting Polymer via a Chiral Small-Molecule Dopant. Adv. Mater. 2013, 25, 2624.

(34) Cao, D.; Liu, Q.; Zeng, W.; Han, S.; Peng, J.; Liu, S. Diketopyrrolopyrrole-Containing Polyfluorenes: Facile Method To Tune Emission Color and Improve Electron Affinity. Macromolecules 2006, 39, 8347.

(35) Langeveld-Voss, B. M. W.; Janssen, R. A. J.; Meijer, E. W. On the origin of optical activity in polythiophenes. J. Mol. Struct. 2000, $521,285$.

(36) Langeveld-Voss, B. M. W.; Christiaans, M. P. T.; Janssen, R. A. J.; Meijer, E. W. Inversion of Optical Activity of Chiral Polythiophene Aggregates by a Change of Solvent. Macromolecules 1998, 31, 6702.

(37) Peet, J.; Kim, J. Y.; Coates, N. E.; Ma, W. L.; Moses, D.; Heeger, A. J.; Bazan, G. C. Efficiency enhancement in low-bandgap polymer solar cells by processing with alkane dithiols. Nat. Mater. 2007, 6, 497. 
(38) Lou, S. J.; Szarko, J. M.; Xu, T.; Yu, L.; Marks, T. J.; Chen, L. X. Effects of Additives on the Morphology of Solution Phase Aggregates Formed by Active Layer Components of High-Efficiency Organic Solar Cells. J. Am. Chem. Soc. 2011, 133, 20661. 